\title{
Estudio Morfométrico de la Arteria Radial y su Implicación en la Cirugía de Revascularización Miocárdica
}

\author{
Morphometric Study of the Radial Artery and its Involvement in Bypass Surgery Grafting \\ Cynthia Guadalupe Reyes Hernández; Sergio Everardo Velázquez Gauna; Juan José Bazaldúa Cruz; \\ José Miguel Hinojosa Amaya; Rodrigo E. Elizondo Omaña \& Santos Guzmán López
}

REYES, H. C. G.; VELÁZQUEZ, G. S. E.; BAZALDÚA, C. J. J.; HINOJOSA, A. J. M.; ELIZONDO-OMAÑA, R. E. \& GUZMÁN, L. S. Estudio morfométrico de la arteria radial y su implicación en la cirugía de revascularización miocárdica. Int. J. Morphol., 31(3):791796, 2013.

RESUMEN: La arteria radial (AR) es utilizada en la práctica médica para la realización de diversos procedimientos quirúrgicos, entre los que destaca la cirugía de revascularización miocárdica. En la literatura actual hay poca información acerca de la compatibilidad de esta arteria con otros conductos vasculares. El objetivo fue determinar las características anatómicas, morfométricas y distribución de la arteria radial en el antebrazo. Se disecó la arteria radial de 10 antebrazos de cadáveres embalsamados; se identificaron y registraron los ramos musculares y vasa nervorum emitidos por la arteria, se midió la longitud total y obtuvieron tres muestras (proximal, media y distal) de cada una para ser procesadas mediante técnicas histológicas y se determinaron los grosores de la túnica media y los diámetros. Se observaron modas de 8 ramos arteriales para el músculo braquiorradial, 4 para los músculos flexor superficial de los dedos y flexor radial del carpo, un ramo arterial único para el músculo pronador cuadrado y una moda de 1 vasa nervorum para el ramo superficial del nervio radial. La longitud total de la arteria fue de $21,94 \mathrm{~cm}( \pm 3,34)$. Los grosores encontrados fueron de $196,16 \mu \mathrm{m}( \pm 72,35), 148,25 \mu \mathrm{m}$ $( \pm 40,40)$ y $158,96 \mu \mathrm{m}( \pm 45,74)$ en los segmentos proximal, medio y distal respectivamente. Los diámetros luminales mostraron una media de $1,48 \mathrm{~mm}( \pm 0,70)$ en la región proximal, $1,01 \mathrm{~mm}( \pm 0,35)$ en la media y $1,43 \mathrm{~mm}( \pm 0,47)$ en la distal. Considerando las características morfométricas, la arteria radial es una opción que satisface los criterios de longitud, diámetro luminal y grosor para ser utilizada como injerto.

PALABRAS CLAVE: Morfometría; Arteria Radial; Cirugía de revascularización miocárdica.

\section{INTRODUCCIÓN}

Cada año a nivel mundial, se realizan cerca de 1 milón de procedimientos quirúrgicos para la revascularización miocárdica en pacientes con cardiopatías isquémicas (Nezic et al., 2006). La cirugía de bypass coronario es el procedimiento más utilizado en enfermedades cardiacas avanzadas. A la fecha se ha demostrado que este tipo de cirugía provee excelentes resultados a corto y mediano plazo; sin embargo los resultados a largo plazo están fuertemente influenciados por el conducto vascular empleado como injerto. De 5 a 7 años después de la cirugía, los pacientes tienen un mayor riesgo de sufrir complicaciones isquémicas relacionadas con el injerto utilizado (Verma et al., 2004).

Los injertos de la vena safena magna (VSM) fueron los primeros que se utilizaron en el bypass coronario (Favaloro, 1968) y en la actualidad siguen siendo una opción para los cirujanos. A pesar de tener un acceso relativamente fácil, de poseer una adecuada longitud y un diámetro correcto; su permeabilidad disminuye con el tiempo (Tatoulis et al., 2002), lo cual se le atribuye a una combinación de hiperplasia en la túnica íntima y a una acelerada aterosclerosis (Verma et al.). Posteriormente comenzaron a utilizarse conductos arteriales (Tatoulis et al., 2004) entre los que destacan las arterias torácica interna izquierda, torácica interna derecha, gastro-omental derecha (AGOD), epigástrica inferior (AEI), radial (AR) y ulnar (AU)(Buxton et al., 2009; HinojosaAmaya et al., 2010). Sin embargo, existen diversos factores que limitan el uso de dichos conductos: longitud insuficiente, diámetro luminal pequeño, fragilidad de la pared vascular y las posibles complicaciones al momento de realizar la extracción de la arteria. Además que algunos de los conductos mencionados no pueden ser extraídos en personas ancianas, con obesidad o en diabéticos (factores de riesgo comunes en pacientes con enfermedades cardiacas) (Verma et al.). 
La AR fue utilizada por primera vez como conducto vascular en la cirugía de bypass coronario por Carpentier en 1971 (Carpentier et al., 1973), pero esta técnica fue abandonada debido a la existencia de un alto rango de oclusión en dicho injerto a los pocos años del postoperatorio. Tiempo después, Acar demostró buenos resultados a largo plazo con el uso de la arteria radial (Acar et al., 1992). Entre las características que hacen de esta arteria un conducto popular para la cirugía de revascularización miocárdica tenemos: el diámetro luminal, la longitud (Sajja et al., 2005), la gruesa pared muscular y su adaptación a altas presiones arteriales (Risteski et al., 2006), además que puede ser extraída en la mayoría de los pacientes (obesos, ancianos, diabéticos) (Tatoulis et al., 2002). Por otra parte también existen desventajas clínicas: es muy propensa a sufrir espasmos (Manabe et al., 2005) y su extracción puede ocasionar complicaciones menores en el antrebrazo y mano (Meharwal \&Trehan, 2001); además en la literatura están reportados cuatro casos de isquemia severa en la mano (Manabe et al.).

Debido al alto porcentaje de complicaciones isquémicas posteriores a la cirugía de revascularización miocárdica y a la estrecha relación con el injerto vascular, es necesario realizar una óptima elección del conducto a utilizar. La elección clínica de dicho conducto se fundamenta en las características específicas del paciente, en las características morfométricas del injerto, en las características anatómicas de la arteria coronaria donde se va a realizar el injerto y en otros factores técnicos (incluyendo técnicas de extracción y uso de fármacos antiespasmódicos) (He, 2006). El objetivo de nuestro estudio fue describir las características morfométricas de la arteria radial.

\section{MATERIAL Y MÉTODO}

Se analizaron las características morfométricas in situ e histológicas de 10 antebrazos de cadáveres embalsamados proporcionados por el Hospital Universitario "Dr. José Eleuterio González".

Estudio in situ. Se disecó la arteria radial en 10 antebrazos de cadáveres embalsamados. Se describió el número de ramos musculares y su distribución, la existencia de vasa nervorum y se midió la longitud total de la arteria. En cuanto a los ramos musculares, se disecaron los ramos que emitía la arteria, se siguió su trayecto, se identificó hacia qué músculo se dirigían, se seccionaron inmediatamente antes de su penetración en el músculo y se registra- ron para su posterior análisis. La existencia de vasa nervorum se consideró cuando un ramo arterial penetraba en el epineuro. Se extrajo la arteria realizando dos cortes: uno proximal y uno distal. El corte proximal se hizo a la altura de la bifurcación de la arteria braquial y dicho punto se consideró el origen de las mediciones posteriores. El corte distal se efectuó a la altura de la apófisis estiloides del radio y se consideró el punto final de las mediciones. La longitud total de la arteria (distancia entre su origen y su fin) se midió con un vernier (cartabón) milimétrico digital con una resolución de $0.01 \mathrm{~mm}$. Esta medida se reportó en centímetros.

Estudio histológico. Se obtuvieron tres muestras de cada arteria: proximal, media y distal. El segmento proximal se tomó del origen de la arteria, el distal del final de la arteria y el medio de la distancia media entre los segmentos proximal y distal. Las muestras fueron procesadas mediante técnicas histológicas y se tiñeron con hematoxilina y eosina. Se verificó que la pared arterial estuviese íntegra y posteriormente se realizó la tinción de Tricrómico de Masson. Se obtuvo la medida del grosor de la túnica media así como el diámetro luminal de los tres segmentos utilizando el programa AxioVs40 V 4.8.1.0 para Windows. Estas medidas se reportaron en $\mu \mathrm{m}$ y mm respectivamente.

Las mediciones fueron analizadas utilizando Microsoft Excel 2010 para Windows. Se reportó la media y la desviación estándar de cada parámetro. Se elaboraron tablas para su representación.

\section{RESULTADOS}

Estudio in situ. La moda de los ramos arteriales para el músculo braquiorradial fue de 8 (3-8), para el músculo flexor radial del carpo de 4 (1-10) y para el músculo flexor superficial de los dedos una moda de 4 (3-5). Para el músculo pronador cuadrado se observó un ramo arterial único. Se identificó una moda de 1 vasa nervorum para el ramo superficial del nervio radial (Tabla I). La longitud promedio de la arteria radial fue de $21,94 \mathrm{~cm}( \pm 3,34)$.

Estudio histológico. La medida del grosor de la túnica media en el segmento proximal fue de $196,16 \mu \mathrm{m}( \pm 72,35)$, en el segmento medio de $148,25 \mu \mathrm{m}( \pm 40,40)$ y en el segmento distal 158,96 $\mu \mathrm{m}( \pm 45,74)$ (Tabla II, Fig. 2). Los diámetros luminales mostraron una media de $1,48 \mathrm{~mm}( \pm 0,70)$ en la región proximal, $1,01 \mathrm{~mm}( \pm 0,35)$ en la media y $1,43 \mathrm{~mm}$ $( \pm 0,47)$ en la dista (Tabla II). 


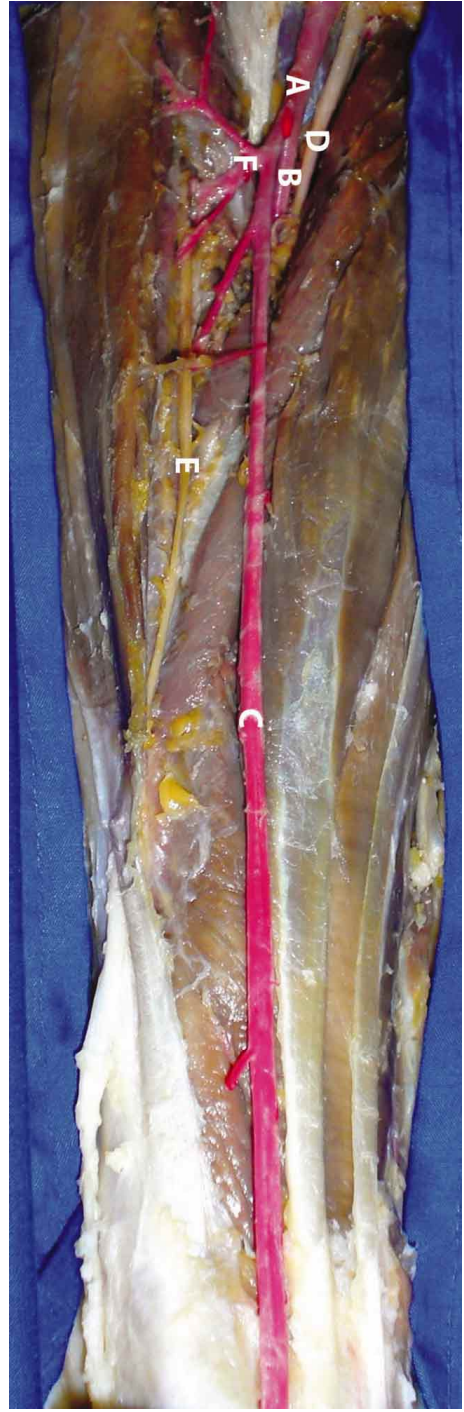

Fig. 1. Disección profunda de compartimento anterior del antebrazo. Estudio in situ: A. Arteria braquial. B. Arteria ulnar. C. Arteria radial. D. Nervio mediano. E. Ramo superficial del nervio radial. F. Ramos para el músculo braquioradial.
Tabla I. Ramos musculares y existencia de vasa nervorum.

\begin{tabular}{llc}
\hline Músculo & Moda & Ramos \\
\hline Braquioradial & 8 & Rango \\
Flexor radial del carpo & 4 & $3-8$ \\
Flexor superficial de los dedos & 4 & $1-10$ \\
Pronador cuadrado & - & $3-5$ \\
Vasa nervorum & - & $0-1$ \\
\hline
\end{tabular}

Tabla II. Grosor de la túnica media y diámetro luminal.

\begin{tabular}{llccc} 
Muestra & \multicolumn{2}{c}{ Grosor $(\mu \mathbf{m})$} & \multicolumn{2}{c}{ Diámetro $(\mathbf{m m})$} \\
Proximal & Media & SD & Media & SD \\
Media & 196.16 & \pm 72.35 & 1.48 & \pm 0.70 \\
Distal & 148.25 & \pm 40.40 & 1.01 & \pm 0.35 \\
& 158.96 & \pm 45.74 & 1.43 & \pm 0.47 \\
\hline
\end{tabular}

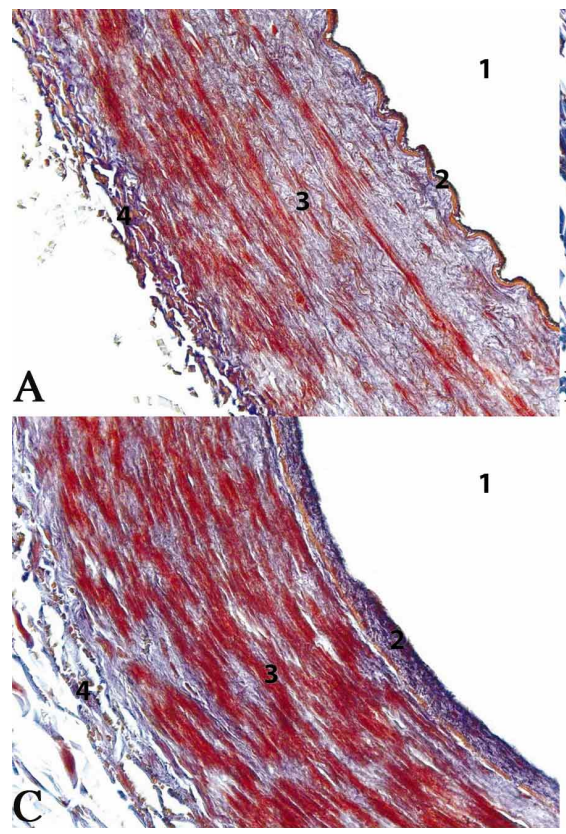

Fig. 2. Microfotografías de corte transversal de arteria radial teñidas con Tricrómico de Masson. Objetivo 40x. Se observa la composición de la túnica media formada principalmente por músculo liso y pocas fibras elásticas A. Segmento proximal. B. Segmento medio. C. Segmento distal. 1. Luz arterial. 2. Túnica íntima. 3. Túnica media. 4. Túnica adventicia.

\section{DISCUSIÓN}

Algunas características anatómicas y morfológicas son indispensables para que una arteria pueda ser utilizada como un conducto en la cirugía de bypass coronario, esto principalmente porque el éxito a largo plazo de dicho procedimiento está fuertemente relacionado con el conducto vascular seleccionado (Verma et al.). Entre tales características se encuentran: longitud del conducto, diámetro luminal, grosor de la pared arterial, viabilidad del conducto y posibles complicaciones isquémicas en el sitio de extracción (Buxton et al.; Hinojosa-Amaya et al.). 
REYES, H. C. G.; VELÁZQUEZ, G. S. E.; BAZALDÚA, C. J. J.; HINOJOSA, A. J. M.; ELIZONDO-OMAÑA, R. E. \& GUZMÁN, L. S. Estudio morfométrico de la arteria radial y su implicación en la cirugía de revascularización miocárdica. Int. J. Morphol., 31(3):791-796, 2013.

Tabla III. Comparación de la longitud total de la arteria radial, grosor de la túnica media y diámetros luminales.

\begin{tabular}{|c|c|c|c|c|c|}
\hline \multirow[b]{2}{*}{ Autor } & \multirow[b]{2}{*}{ Longitud (cm) } & \multirow[b]{2}{*}{ Proximal (mm) } & \multirow[b]{2}{*}{ Medio (mm) } & \multicolumn{2}{|c|}{ Diámetros } \\
\hline & & & & Distal (mm) & Único $(\mathbf{m m})$ \\
\hline Bhan et al., 1999 & $15-25$ & - & - & - & - \\
\hline Brodman et al., 2002 & - & - & - & - & $4.29 \pm 1.22$ \\
\hline Elizondo et al., 2003 & $21.57 \pm 4.42$ & $4.25 \pm 0.5$ & - & $3.25 \pm 0.5$ & - \\
\hline Haerle et al., 2003 & - & $3.5 \pm 0.3$ & - & $3.3 \pm 0.2$ & - \\
\hline Riekkinen et al., 2003 & - & - & - & - & $3 \pm 0.5$ \\
\hline Verma et al., 2004 & $>20$ & - & - & - & $2-3$ \\
\hline Gaudino et al., 2005 & - & - & - & - & $2.14 \pm 0.52$ \\
\hline Guo-Wei, 2006 & 20.5 & - & - & - & - \\
\hline Risteski et al., 2006 & $18-22$ & - & - & - & $2-3$ \\
\hline Massey et al., 2007 & $19.1 \pm 0.9$ & - & - & - & $3.1 \pm 0.3$ \\
\hline Ryszawa et al., 2007 & $>20$ & - & - & - & $2-3$ \\
\hline Ohonen et al., 2008 & - & $3.02 \pm 0.9$ & - & $2.4 \pm 0.65$ & - \\
\hline Brzezinski et al, 2009 & - & - & - & - & 2.45 \\
\hline Cagli et al., 2006 & - & - & - & - & $2.4 \pm 0.4$ \\
\hline Est udio actual & $21.94 \pm 3.34$ & $1.48 \pm 0.70$ & $1.01 \pm 0.35$ & $1.43 \pm 0.47$ & - \\
\hline
\end{tabular}

La extracción de la arteria radial puede ocasionar algunas complicaciones en el antebrazo y la mano de donde se extrajo. Las complicaciones más comunes son anormalidades isquémicas y sensoriales. En la literatura existen 4 casos reportados de isquemia severa en la mano (Manabe et al.), debido a esto es necesario conocer los ramos musculares emitidos por la arteria radial. ElizondoOmaña et al. (2003) describieron el territorio muscular irrigado por la arteria radial, en donde el músculo que recibía una mayor irrigación proporcionada por dicha arteria era el braquiorradial, lo cual apoya a los resultados obtenidos en el presente estudio. Previo a la extracción de la arteria radial deben realizarse algunos estudios para conocer si existe una adecuada circulación colateral y de esta manera evitar las posibles complicaciones isquémicas. En las anormalidades sensoriales se encuentran principalmente parestesias cutáneas en la distribución de los nervios cutáneo lateral del antebrazo y ramo superficial del nervio radial (Nezic et al.). La existencia de vasa nervorum emitida por la arteria radial no había sido reportada en la literatura, en el presente estudio se encontró una moda de uno para el ramo superficial del nervio radial. Puesto que las complicaciones que más se presentan son las sensoriales es pertinente realizar más estudios sobre la presencia de vasa nervorum emitida por la arteria radial.

La longitud del conducto vascular seleccionado es importante para establecer con cuál arteria coronaria se va a realizar la anastomosis. Según He, la arteria radial posee una adecuada longitud para anastomosarse con cualquier rama de las arterias coronarias, incluso puede dividirse en dos segmentos para formar dos injertos independientes o secuencias para múltiples anastomosis distales
(González Santos et al., 2005). Estudios previos describen que la arteria radial comúnmente se anastomosa con las arterias circunfleja e interventricular posterior (Tatoulis et al., 2004; Sajja et al.; Hinojosa-Amaya et al.). En el actual estudio la media de la longitud total encontrada coincide con las obtenidas en otros estudios (Tabla III), dicha medida hace de la arteria radial un conducto ideal para realizar cualquier tipo de anastomosis.

El grosor de la pared vascular es importante al momento de considerar el sitio en el cual se va colocar el injerto. En la actualidad la mayor parte de los estudios miden el grosor de la pared vascular solamente en un segmento de la arteria, la medida única del grosor de la túnica media obtenida por González Santos et al., es superior al doble del mayor grosor obtenido en nuestro estudio. Debido a que la arteria radial puede ser utilizada para injertos independientes, en nuestro estudio consideramos importante medir el grosor de la pared en tres regiones de la arteria.

El diámetro luminal de la arteria radial es muy cercano al reportado en la circulación coronaria, lo cual facilita su anastomosis con la aorta y con las arterias coronarias (Verma et al.). En la literatura hay varios estudios que describen el diámetro de la arteria radial; sin embargo la mayoría de éstos miden un diámetro único, en el presente estudio el diámetro luminal se midió en tres segmentos (Tabla III). Tanto el grosor como el diámetro luminal son importantes al momento de elegir el sitio de anastomosis distal, esto porque la gruesa pared muscular de la arteria radial le permite ser utilizada como injerto en arterias donde la oclusión de la luz es mayor al $70 \%$. 
En conclusión, al considerar las características morfométricas, la arteria radial es una opción que satisface los criterios de longitud, diámetro luminal y grosor para ser utilizada como injerto vascular en la cirugía de revascularización miocárdica.
AGRADECIMIENTOS. A todo el personal que conforma los departamentos de Anatomía Humana, Embriología e Histología de la Facultad de Medicina de la U.A.N.L. por su constante apoyo, soporte y confianza.

REYES, H. C. G.; VELÁZQUEZ, G. S. E.; BAZALDÚA, C. J. J.; HINOJOSA, A. J. M.; ELIZONDO-OMAÑA, R. E. \& GUZMÁN, L. S. Morphometric study of the radial artery and its involvement in bypass surgery grafting. Int. J. Morphol., 31(3):791-796, 2013.

SUMMARY: The radial artery (RA) is used in medical practice to perform different surgical procedures, of which most realized is the coronary artery bypass graft surgery. In the current literature there is little information about the compatibility of this artery with other vascular conduits. Therefore the aim of this study was to determine the anatomical, morphometric and distribution characteristics of radial artery on the forearm. Radial artery was dissected from the forearms of 10 embalmed cadavers, muscular branches and vasa nervorum were identified and registered, total length was measured. Three samples of each artery were obtained (proximal, middle and distal) to be processed by histological techniques and to determine the thickness of its tunica media and its diameters. The results show a mode of 8 arterial branches to the brachioradialis, a mode of 4 for the superficial flexor muscles of the fingers and flexor carpi radialis, we found an unique arterial branch to the pronator quadratus and a mode of 1 vasa nervorum of superficial branch of radial nerve. The total length of the artery was $21.94 \mathrm{~cm}( \pm 3.34)$. The vascular wall thickness we found were $196.16 \mathrm{~mm}( \pm 72.35), 148.25 \mathrm{~mm}( \pm 40.40)$ and $158.96 \mathrm{~mm}( \pm 45.74)$ in the proximal, middle and distal segments respectively. The luminal diameters showed an average of $1.48 \mathrm{~mm}$ $( \pm 0.70)$ in the proximal region, $1.01 \mathrm{~mm}( \pm 0.35)$ in the middle region and $1.43 \mathrm{~mm}( \pm 0.47)$ in the distal region. In the present study we concluded that considering the morphometric characteristics, the radial artery is an option that satisfied the criteria of length, thickness and luminal diameter to be used as a graft in the coronary artery bypass graft surgery.

KEY WORDS: Morphometry; Radial artery; Coronary artery bypass grafting.

\section{REFERENCIAS BIBLIOGRÁFICAS}

Acar, C.; Jebara, V. A.; Portoghese, M.; Beyssen, B.; Pagny, J. Y.; Grare, P.; et al. Revival of the radial artery for coronary artery bypass grafting. Ann. Thorac. Surg., 54(4):652-660, 1992.

Bhan, A.; Gupta, V.; Choudhary, S. K.; Sharma, R.; Singh, B.; Aggarwal, R.; et al. Radial artery in CABG: could the early results be comparable to internal mammary artery graft? Ann. Thorac. Surg., 67(6):1631-6, 1999.

Brodman, R. F.; Hirsh, L. E. \&Frame, R. Effect of radial artery harvest on collateral forearm blood flow and digital perfusion. J. Thorac. Cardiovasc. Surg., 123(3):512-6, 2002.

Brzezinski, M.; Luisetti, T. \&London, M. J.Radial artery cannulation: a comprehensive review of recent anatomic and physiologic investigations.Anesth. Analg., 109(6):1763-81, 2009.

Buxton, B. F.; Hayward, P. A.; Newcomb, A. E.; Moten, S.; Seevanayagam, S. \& Gordon, I. Choice of conduits for coronary artery bypass grafting: craft or science? Eur. J. Cardiothorac. Surg., 35(4):658-70, 2009.

Cagli, K.; Uzun, A.; Emir, M.; Bakuy, V.; Ulas, M. \&Sener, E. Correlation of modified a Allen test with Doppler ultrasonography. Asian Cardiovasc. Thorac. Ann., 14(2):1058, 2006.
Elizondo-Omaña, R. E.; Herrera, J. A.; Leal, E. M.; Hernández, I.; Guzmán, A.; Sánchez, A.; et al. Comparación anatómica de la arteria torácica interna con la arteria radial. Med. Univer., 5(19):83-6, 2003.

Favaloro, R. G. Saphenus vein autograft replacement of severe segmental coronary artery occlusion: operative technique. Ann. Thorac. Surg., 5(4):334-9, 1968.

Gaudino, M.; Prati, F.; Caradonna, E.; Trani, C.; Burzotta, F.; Schiavoni, G.; et al. Implantation in coronary circulation induces morphofunctional transformation of radial grafts from muscular to elastomuscular. Circulation, 112(9 Suppl):I20811, 2005.

González Santos, J. M.; López Rodríguez, J. \& Dalmau Sorlí, M. J. Los injertos arteriales en cirugía coronaria: ¿una terapia universal? Rev. Esp. Cardiol., 58(10):1207-23, 2005.

Haerle, M.; Häfner, H. M.; Dietz, K.; Schaller, H. E. \&Brunelli, F. Vascular dominance in the forearm. Plast. Reconstr. Surg., 111(6):1891-8, 2003.

He, G. W. Considerations in the choice of arterial grafts.Arterial Grafting for Coronary Artery Bypass Surgery. Heidelberg,Springer Berlin Heidelberg, 2006. pp.81-6. 
REYES, H. C. G.; VELÁZQUEZ, G. S. E.; BAZALDÚA, C. J. J.; HINOJOSA, A. J. M.; ELIZONDO-OMAÑA, R. E. \& GUZMÁN, L. S. Estudio morfométrico de la arteria radial y su implicación en la cirugía de revascularización miocárdica. Int. J. Morphol., 31(3):791-796, 2013.

Hinojosa-Amaya, J. M.; Villarreal-Silva, E. E.; Elizondo-Omaña, R. E.; Gutiérrez de la O., J.; Dávila-Canales, R.; GonzálezCantú, A.; et al. Conduits for myocardial revascularization grafts: The importance of morphology and imaging. Med. Univer., 12:115-9, 2010.

Kohonen, M.; Teerenhovi, O.; Terho, T.; Laurikka, J. \&Tarkka, M. Non-harvestable radial artery. A bilateral problem? Interact. Cardiovasc. Thorac. Surg., 7(5):797-800, 2008.

Manabe, S.; Tabuchi, N.; Tanaka, A. H.; Arai, H. \& Sunamori, M. Hand circulation after radial artery harvest for coronary artery bypass grafting. J. Med. Dent. Sci., 52(2):101-7, 2005.

Massey, R. M.; Warren, O. J.; Szczeklik, M.; Wallace, S.; Leff, D. R.; Kokotsakis, J.; et al. Skeletonization of radial and gastroepiploic conduits in coronary artery bypass surgery. $J$. Cardiothorac. Surg., 2:26, 2007.

Meharwal, Z. S. \&Trehan, N. Functional status of the hand after radial artery harvesting: results in 3,977 cases. Ann. Thorac. Surg., 72(5):1557-61, 2001.

Nezic, D. G.; Knezevic, A. M.; Milojevic, P. S.; Dukanovic, B. P.; Jovic, M. D., Borzanovic, M. D.; et al. The fate of the radial artery conduit in coronary artery bypass grafting surgery. Eur. J. Cardiothorac. Surg., 30(2):341-6, 2006.

Riekkinen, H. V.; Karkola, K. O. \& Kankainen, A. The radial artery is larger than the ulnar. Ann. Thorac. Surg., 75(3):882-4, 2003.

Risteski, P. S.; Akbulut, B.; Moritz, A. \&Aybek, T.The radial artery as a conduit for coronary artery bypass grafting: review of current knowledge. Anadolu Kardiyol. Derg., 6(2):153-62, 2006.

Ryszawa, N.; Rudzin’ski, P.; Piatek, J.; Mrózek, B.; Krawiec, P.; Kosiniak-Kamysz, W.; et al. Different reactivity of the proximal and distal segments of the radial artery to vasoconstrictors in patients undergoing coronary artery bypass grafting. Kardiol. Pol., 65(11):1313-9; discussion1320, 2007.

Sajja, L. R.; Mannam, G.;Pantula, N. R. \&Sompalli, S.Role of Radial Artery Graft in Coronary Artery Bypass Grafting. Ann. Thorac. Surg., 79(6):2180-8, 2005.

Tatoulis, J.; Buxton, B. F. \& Fuller, J. A. Patencies of 2,127 arterial to coronary conduits over 15 years. Ann. Thorac. Surg., 77(1):93-101, 2004.

Tatoulis, J.; Royse, A. G.; Buxton, B. F.; Fuller, J. A.; Skillington, P. D.; Goldblatt, J. C.; et al. The radial artery in coronary surgery: a 5-year experience-clinical and angiographic results. Ann. Thorac. Surg., 73(1):143-8, 2002.

Verma, S.; Szmitko, P. E.; Weisel, R. D.; Bonneau, D.; Latter, D.; Errett, L.; et al. Should radial arteries be used routinely for coronary artery bypass grafting? Circulation, 110(5):e40-6, 2004.

\author{
Dirección para Correspondencia: \\ Dr. Rodrigo E. Elizondo-Omaña \\ Departamento de Anatomía Humana \\ Facultad de Medicina \\ Universidad Autónoma de Nuevo León (UANL) \\ Ave. Madero s/n Col. Mitras Centro \\ Monterrey, N.L. C.P.64460 \\ MEXICO
}

Tel: 01 (52) 8183294171

Email: rod_omana@yahoo.com

Recibido : 18-10-2012

Aceptado: 29-04-2013 